\title{
Group Search Optimizer for the Mobile Location Management Problem
}

\author{
Dan Wang, ${ }^{1}$ Congcong Xiong, ${ }^{1}$ and Wei Huang ${ }^{2,3}$ \\ ${ }^{1}$ School of Computer Science and Information Engineering, Tianjin University of Science \& Technology, Tianjin 300222, China \\ ${ }^{2}$ State Key Laboratory of Virtual Reality Technology and Systems, Beihang University, Beijing 100191, China \\ ${ }^{3}$ School of Computer and Communication Engineering, Tianjin University of Technology, Tianjin 300384, China
}

Correspondence should be addressed to Wei Huang; huangwabc@163.com

Received 30 May 2014; Revised 18 July 2014; Accepted 25 July 2014; Published 11 August 2014

Academic Editor: Wanneng Shu

Copyright (C) 2014 Dan Wang et al. This is an open access article distributed under the Creative Commons Attribution License, which permits unrestricted use, distribution, and reproduction in any medium, provided the original work is properly cited.

\begin{abstract}
We propose a diversity-guided group search optimizer-based approach for solving the location management problem in mobile computing. The location management problem, which is to find the optimal network configurations of management under the mobile computing environment, is considered here as an optimization problem. The proposed diversity-guided group search optimizer algorithm is realized with the aid of diversity operator, which helps alleviate the premature convergence problem of group search optimizer algorithm, a successful optimization algorithm inspired by the animal behavior. To address the location management problem, diversity-guided group search optimizer algorithm is exploited to optimize network configurations of management by minimizing the sum of location update cost and location paging cost. Experimental results illustrate the effectiveness of the proposed approach.
\end{abstract}

\section{Introduction}

Mobile location management becomes more and more important with the rapid growing mobile network in recent years. Lots of novel applications for data transfer based on fax, voice, and many other mobile services need to be taken into account for making the next generation of mobile network, which supports a basic global personal communication network. To support such applications, mobility management has to be considered when designing infrastructure for wireless mobile networks [1-4].

Reporting cells scheme is conventionally a kind of location management strategies for implementing effective location management [5]. In this case, the minimization of location management cost is considered to decide the reporting cells configuration and finding the optimal set of the reporting cells can be regarded as an optimization problem. However, it is the fact that such an optimization problem has been proven an NP-complete problem [6]. To address this problem, many optimization approaches have been proposed in the past decades. Pioneering work by Taheri and Zomaya $[7,8]$, Demestichas et al. [9], and Yuen and Wong [10] has studied the mobile location management problem using different evolutionary optimization approaches. In a nutshell, evolutionary algorithms have proven to be very useful for optimizing such problems.

Recently, group search optimizer (GSO) [11] inspired by animal behavior is proposed as a new evolutionary algorithm, and it obtains effective competitive performance when solving some optimization problems $[12,13]$. However, such animal behavior-inspired algorithm is essentially stochastic search techniques like many other evolutionary algorithms. Such random mechanism leads to premature convergence problem due to purely random resampling or selections of individuals from a given population, which may lead to revisiting nonproductive regions of the search space [12]. This drawback sometimes limits the application of GSO when dealing with online problems or real-time constraints.

In this paper, we propose a diversity-guided group search optimizer (DGSO) to deal with the location management in mobile computing. In the design of DGSO, diversity guidance is used to prevent the fact that the reducing diversity takes place too early. With the use of diversity guidance, selection 


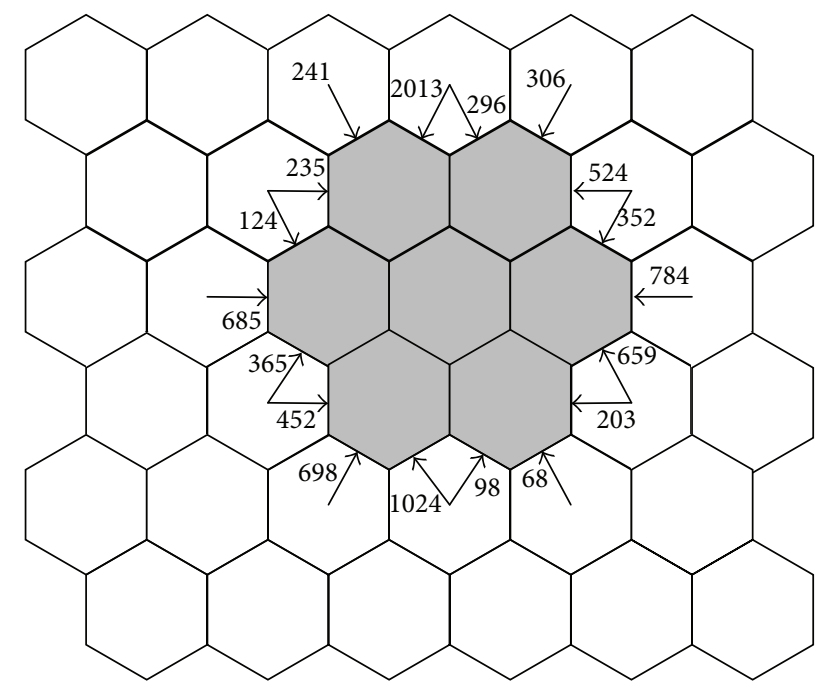

FIGURE 1: Entering flow of users and call arrival.

operator keeps the diversity of population. Moreover, the proposed DGSO is employed to optimization of the set of the reporting cells in the mobile location management problem.

\section{Mobile Location Management Problem}

In cellular network systems, the mobile location management problem consists of two basic operations, namely, location update and location inquiry. The location update corresponds to the notification of current location, performed by mobile terminals when they change their location in the network, while the location inquiry is the operation of determining the location of the mobile terminal, which is executed by the network when it tries to direct an incoming call to the user [5].

As to mobile location management problem, here we consider minimizing the location management cost, which includes location update operation and location paging operation.

The location update cost (LUC) corresponds to the cost of user which performs an update when it moves from the current location area into another location area. If we consider a typical GSM network as shown in Figure 1, it is easy to see the total number of users that enter in the white location area. The location update cost can be calculated as the following expression:

$$
\begin{aligned}
N_{\mathrm{LUC}}= & 2013+296+306+524+352+784+659 \\
& +203+68+98+1024+698+452 \\
& +365+685+124+235+241=9127 .
\end{aligned}
$$

The location paging operation cost is caused by the network when it tries to locate a user's mobile terminal, during the location inquiry, and normally the number of

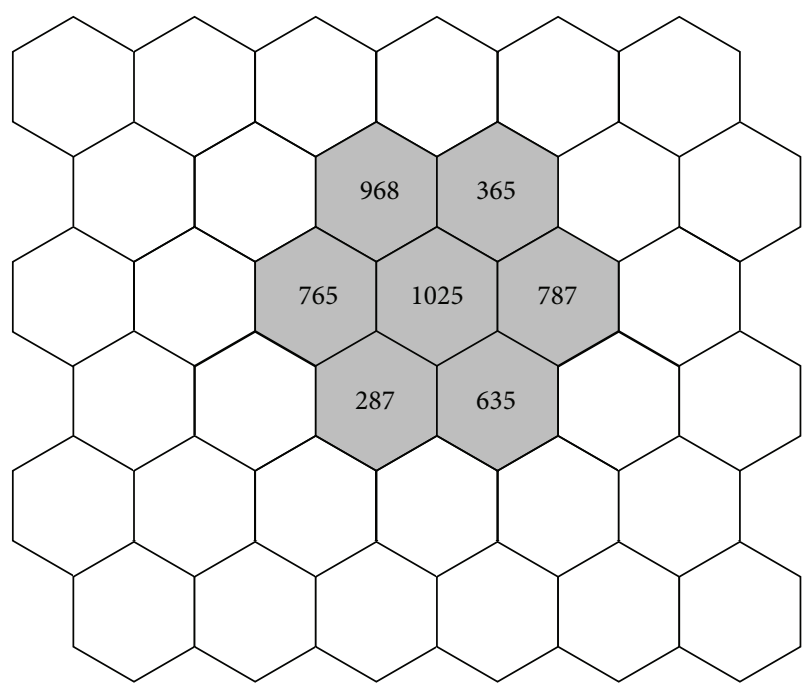

FIGURE 2: Entering flow of users for an instant locafiguretion area.

paging transactions is directly related to the number of incoming calls [7]. Figure 2 shows an example of incoming calls to the white location area. The paging cost can be obtained as follows:

$$
N_{P}=968+365+765+1025+787+287+635=4832 .
$$

Generally, the total cost of location areas is computed by using the following formula [7]:

$$
\text { Cost }=\beta \times N_{\mathrm{LUC}}+N_{P}
$$

The cost of a location update is normally considered to be 10 times greater than the cost of paging; that is, $\beta=10$ $[1,14-16]$. With the combination of location update cost (1) and location paging costs (2), the total cost by (3) can be calculated as follows:

$$
\text { Cost }=10 \times 9127+4832=96102 .
$$

In the reporting cells scheme the location updates only are preformed when a mobile user enters in a reporting cell and the vicinity factor of each cell must be considered [1]. In this case, the genetic formula given by (3) must be readjusted and it is formulated as [15]

$$
\text { Cost }=\beta \times \sum_{i \in S} N_{\mathrm{LUC}}(i)+\sum_{i=0}^{N} N_{P}(i) \times V(i),
$$

where $N$ is the number of location updates related to the reporting cell $i, S$ represents the subset of reporting cells, $N_{p}$ is the number of incoming calls of cell $i, N$ is the number of cells that compound the mobile network configuration, and $V$ is the vicinity factor attributed for cell $i$. 


\section{Group Search Optimizer Algorithm}

Like the swarm intelligence evolutionary algorithms, GSO is also a population-based optimization algorithm. The population is called a group and each individual in the population is called a member $[17,18]$.

3.1. Main Operators in GSO. The GSO algorithm that is based on the producer-scrounger (PS) model has three basic operators. The main operators in GSO algorithm are as follows.

3.1.1. Initialization. For convenience, the related symbols are summarized as follows [19]: in a $n$-dimension search space, the $i$ th member at the $k$ th iteration has a current position $X_{i}^{k} \in \mathbb{R}^{n}$; a head angle $\varphi_{i}^{k}=\left(\varphi_{i_{1}}^{k}, \ldots, \varphi_{i_{(n-1)}}^{k}\right) \in \mathbb{R}^{n-1}$, and a search direction of the $i$ th member which is a unit vector $D_{i}^{k}\left(\varphi_{i}^{k}\right)=\left(d_{i_{1}}^{k}, \ldots, d_{i_{n}}^{k}\right) \in \mathbb{R}^{n}$; the unit vector can be calculated as [20]

$$
\begin{aligned}
& d_{i_{1}}^{k}=\prod_{q=1}^{n-1} \cos \left(\varphi_{i_{q}}^{k}\right) \\
& d_{i_{j}}^{k}=\sin \left(\varphi_{i_{(j-1)}}^{k}\right) \prod_{q=j}^{n-1} \cos \left(\varphi_{i_{q}}^{k}\right) \quad(j=2, \ldots, n-1) \\
& d_{i_{n}}^{k}=\sin \left(\varphi_{i_{(n-1)}}^{k}\right),
\end{aligned}
$$

where the $X_{i}^{k}, \varphi_{i}^{k}$, and $d_{i}^{k}$ are randomly generated for the initialization of group.

For simplifying the algorithm computational capability, we assume that there is only one producer (here we select the best member as the producer) at each iteration for calculating the group member's fitness value.

3.1.2. Producing. When the only producer is selected, it scans the environment for searching the resource. In the GSO algorithm [18], the scanning field was characterized by the maximum pursuit angle $\theta_{\max }$ and the maximum pursuit distance $l_{\max }$. The $k$ th iteration of the $X_{p}$ behaves as follows.

First, the producer will randomly scan three points for getting the best point [21]: at zero degree, the point represents as follows:

$$
X_{z}=X_{p}^{k}+r_{1} l_{\max } D_{p}^{k}\left(\varphi^{k}\right) .
$$

In the right hand side hypercube, the point is expressed as follows:

$$
X_{r}=X_{p}^{k}+r_{1} l_{\max } D_{p}^{k}\left(\varphi^{k}+\frac{r_{2} \theta_{\max }}{2}\right)
$$

In the left hand hypercube, the point represents as follows:

$$
x_{l}=X_{p}^{k}+r_{1} l_{\max } D_{p}^{k}\left(\varphi^{k}-\frac{r_{2} \theta_{\max }}{2}\right),
$$

where $r_{1}$ is the normally distributed random number and $r_{2}$ is the distributed random sequence in the range between 0 and 1.

Second, the producer will find the best point by calculating the fitness value. If the producer cannot find the better resource than the current position, the current position is considered the best point; otherwise, the current point will be changed, and the new angle is generated by using the following formula:

$$
\varphi^{k+1}=\varphi^{k}+r_{2} \alpha_{\max }
$$

where $\alpha_{\max }$ denotes the maximum turning angle.

Assuming that it could not find the better resource than the current position after the ath iterations, we have the angle:

$$
\varphi^{k+a}=\varphi^{k} .
$$

3.1.3. Scrounging. Randomly select $80 \%$ from the rest of members to perform scrounging.

3.1.4. Dispersion. In the disperse operator, we use the random walks [22-24] for searching the distributed resources. At the $k$ th iteration, it generates a random head angle $\varphi_{i}$ using (5); it chooses a random distance

$$
l_{i}=a \cdot r_{1} l_{\max }
$$

and the new point is

$$
X_{i}^{k+1}=X_{i}^{k}+l_{i} D_{i}^{k}\left(\varphi^{k+1}\right) .
$$

3.2. Diversity-Guided GSO. Like the most of populationbased algorithms, the initialization group (population) and the searching operators are started with random guesses due to the lack of a priori information. To alleviate this problem, diversity guidance is explored here for increasing the diversity of initialization group and the generation jumping embedded in the GSO algorithm. Here the variable "diversity" of group (population) [25] is calculated as follows:

$$
\text { diversity }=\frac{1}{|S| \cdot|L|} \cdot \sum_{i=1}^{|S|} \sqrt{\sum_{j=1}^{N}\left(p_{i j}-\bar{p}_{j}\right)^{2}}
$$

where $S$ is the group (population), $|S|$ is the size of group, $|L|$ is the length of longest the diagonal in the search space, $N$ is the dimension of the problem, $p_{i j}$ is the $j$ th value of the $i$ th individual, and $\bar{p}_{j}$ is the $j$ th value of the average point $\bar{p}$; it has also stressed that the diversity measure is independent of group size, the dimensionality of the problem, and the search range in each dimension. For convenience, in this paper $w_{1}$, 
/* Diversity-Guided GSO (DGSO) */

(1) BEGIN

(2) Initialization (Generate uniformly distributed random population);

(3) While the termination condition isn't satisfied

(4) Diversity-Guided Operator;

(5) Producing;

Scrounging;

(6) End While

Dispersion;

(7) END

Algorithm 1: Procedure of diversity-guided GSO (DGSO).

TABLE 1: Cells attribute for the $4 \times 4$ test network.

\begin{tabular}{lccccccccccc}
\hline Cells & $w_{m i}$ & $w_{c i}$ & Cells & $w_{m i}$ & $w_{c i}$ & Cells & $w_{m i}$ & $w_{c i}$ & Cells & $w_{m i}$ & $w_{c i}$ \\
\hline 1 & 518 & 517 & 5 & 1617 & 642 & 9 & 445 & 251 & 13 & 307 & 25 \\
2 & 774 & 573 & 6 & 472 & 951 & 10 & 2149 & 224 & 14 & 385 \\
3 & 153 & 155 & 7 & 650 & 526 & 11 & 1658 & 841 & 15 & 1346 \\
4 & 1696 & 307 & 8 & 269 & 509 & 12 & 952 & 600 & 16 & 572 & 225 \\
\hline
\end{tabular}

TABLE 2: Results for the $4 \times 4$ network.

\begin{tabular}{|c|c|c|c|c|c|c|}
\hline Algorithms & Average value & Deviation & Minimum value & Deviation & Maximum value & Deviation \\
\hline GA [26] & 12.253 & $0.006 \%$ & 12.252 & $0.000 \%$ & 12.373 & $0.986 \%$ \\
\hline TS [26] & 12.252 & $0.000 \%$ & 12.252 & $0.000 \%$ & 12.252 & $0.000 \%$ \\
\hline $\mathrm{AC}[26]$ & 12.252 & $0.000 \%$ & 12.252 & $0.000 \%$ & 12.252 & $0.000 \%$ \\
\hline TDE [27] & 12.252 & $0.000 \%$ & 12.252 & $0.000 \%$ & 12.252 & $0.000 \%$ \\
\hline HCDE [27] & 12.252 & $0.000 \%$ & 12.252 & $0.000 \%$ & 12.252 & $0.000 \%$ \\
\hline \multicolumn{7}{|l|}{ Our methods } \\
\hline GSO & 12.252 & $0.000 \%$ & 12.252 & $0.000 \%$ & 12.252 & $0.000 \%$ \\
\hline DGSO & 12.252 & $0.000 \%$ & 12.252 & $0.000 \%$ & 12.252 & $0.000 \%$ \\
\hline
\end{tabular}

$w_{2}$ are set as 0.33 and 0.66 , respectively. The procedure of diversity-guided operator is realized as follows.

Case I. If diversity is less than dLow, set $l_{\max }=w_{1} * l_{\max }$.

Case II. If diversity is greater than dHigh, do nothing $\left(l_{\max }=\right.$ $\left.l_{\max }\right)$.

Case III. If diversity is greater than or equal to dLow, and diversity is less than dHigh, set $l_{\max }=w_{2} * l_{\max }$.

Here $l_{\max }$ is defined in formula (2), (3), and (4); dLow, dHigh, $w_{1}$, and $w_{2}$ are given numbers.

Furthermore, the procedure of DGSO can be outlined as shown in Algorithm 1.

\section{Experimental Studies}

This section reports the experimental results of the proposed DGSO for solving the location management problem in mobile computing. We compared the performance of DGSO with the performance achieved by some "common" algorithms [8] such as genetic algorithm, tabu search, and the ant colony algorithm, respectively. All algorithms are tested based on three test networks with different sizes, and each algorithm is run 200 times for each test network. Furthermore, to compare with the results reported in the literatures, the cost per call arrival [26] divided the total cost by the total number of call arrivals is used as the fitness (objective value).

The parameters of DGSO and GSO are as follows [8]: the initialization of population is generated uniformed at random; the initial head angle is set to be $\pi / 4$; the maximum pursuit angle $\theta_{\max }$ is $\pi / a^{2}$, where $a$ is given by $\operatorname{Round}(\sqrt{n+1})$ and $n$ is the dimension of solutions; the maximum turning angle is $\theta_{\max } / 2$.

4.1. Test Network with 16 Cells. The first experiment is implemented based on a $4 \times 4$ size network, whose data set is shown in Table 1 [26]. Here the $w_{m i}$ and $w_{c i}$ denote a movement weight and call arrival weight for the cell $i$, respectively.

The algorithm presented earlier is adapted here to solve the problem. Figure 3 shows the process of optimization with the successive generations by using the GSO and DGSO, respectively. Table 2 summarizes the comparison results of running the algorithms. It is evident that the proposed DGSO and GSO lead to smaller mean value in comparison with the 
TABLE 3: Cells attribute for the $6 \times 6$ test network.

\begin{tabular}{lccccccccccc}
\hline Cells & $w_{m i}$ & $w_{c i}$ & Cells & $w_{m i}$ & $w_{c i}$ & Cells & $w_{m i}$ & $w_{c i}$ & Cells & $w_{m i}$ & $w_{c i}$ \\
\hline 1 & 1039 & 714 & 10 & 296 & 221 & 19 & 1945 & 462 & 28 & 1342 & 370 \\
2 & 1476 & 120 & 11 & 793 & 856 & 20 & 1368 & 682 & 29 & 814 & 721 \\
3 & 262 & 414 & 12 & 317 & 652 & 21 & 1850 & 241 & 30 & 747 & 769 \\
4 & 442 & 639 & 13 & 507 & 238 & 22 & 1131 & 700 & 31 & 146 & 17 \\
5 & 1052 & 419 & 14 & 603 & 964 & 23 & 236 & 23 & 32 & 904 & 265 \\
6 & 1902 & 332 & 15 & 1479 & 789 & 24 & 1622 & 827 & 33 & 359 & 958 \\
7 & 444 & 494 & 16 & 756 & 457 & 25 & 16 & 328 & 34 & 1729 & 191 \\
8 & 1103 & 810 & 17 & 695 & 708 & 26 & 332 & 255 & 35 & 190 & 551 \\
9 & 1829 & 546 & 18 & 356 & 825 & 27 & 1203 & 393 & 36 & 1907 & 467 \\
\hline
\end{tabular}

TABLE 4: Results for the $6 \times 6$ network.

\begin{tabular}{|c|c|c|c|c|c|c|}
\hline Algorithms & Average value & Deviation & Minimum value & Deviation & Maximum value & Deviation \\
\hline GA [26] & 11.511 & $0.343 \%$ & 11.471 & $0.000 \%$ & 12.030 & $4.867 \%$ \\
\hline TS [26] & 11.471 & $0.000 \%$ & 11.471 & $0.000 \%$ & 11.471 & $0.000 \%$ \\
\hline $\mathrm{AC}[26]$ & 11.472 & $0.007 \%$ & 11.471 & $0.000 \%$ & 11.573 & $0.883 \%$ \\
\hline TDE [27] & 11.471 & $0.000 \%$ & 11.471 & $0.000 \%$ & 11.471 & $0.000 \%$ \\
\hline HCDE [27] & 11.471 & $0.000 \%$ & 11.471 & $0.000 \%$ & 11.471 & $0.000 \%$ \\
\hline \multicolumn{7}{|l|}{ Our methods } \\
\hline GSO & 11.456 & $0.853 \%$ & 11.426 & $0.000 \%$ & 11.471 & $0.289 \%$ \\
\hline DGSO & 11.432 & $0.676 \%$ & 11.426 & $0.000 \%$ & 11.471 & $0.795 \%$ \\
\hline
\end{tabular}

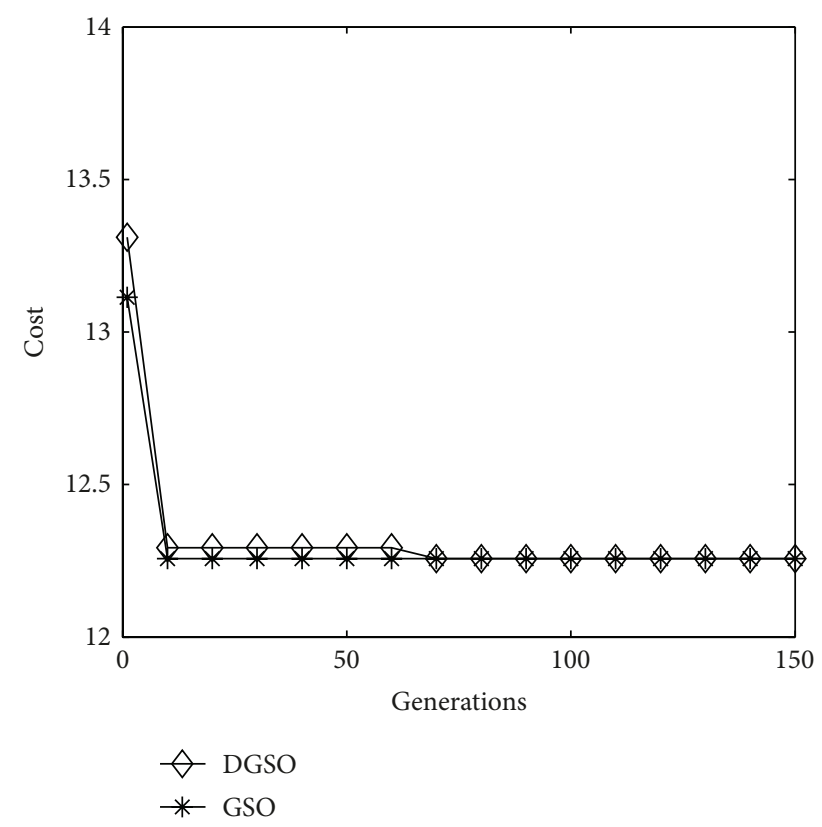

FIgURE 3: Performance index in successive generations $(4 \times 4$ network).

GA; however, the two GSOs obtain the same results as the AC, TDE, and HCDE. That is, the above five algorithms obtain the optimal solution with reporting cells configuration shown in Figure 4.

4.2. Test Network with 36 Cells. For the second experiment, a $6 \times 6$ size network is utilized, and Table 3 provides the data

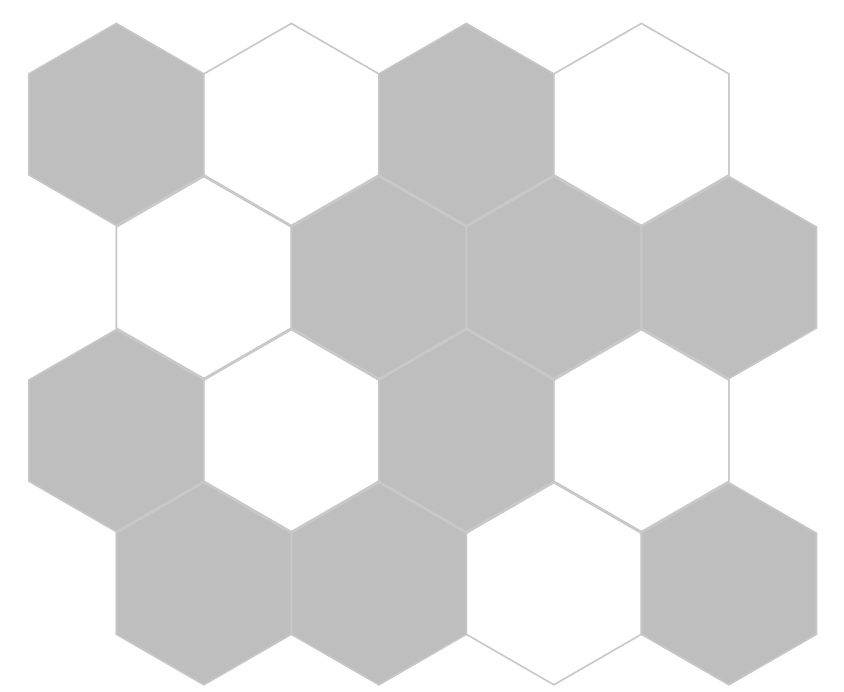

FIGURE 4: $4 \times 4$ test network optimal solution with reporting cells configuration.

set [26]. After running the GSOs with 150 generations, we get the comparison of GSO and DGSO shown in Figure 5. It shows that both DGSO and GSO have low cost and rapid convergence. The comparison results of some common algorithms are summarized in Table 4.

As shown in Table 4, the minimization cost of DGSO is only 11.425 , while the best optimal cost reported in previous literatures is 11.471. It appears that the proposed DGSO outperforms several previous algorithms known in the literature. Figure 6 depicts the optimal solution with reporting cells configuration. 
TABLE 5: Cells attribute for the $8 \times 8$ test network.

\begin{tabular}{|c|c|c|c|c|c|c|c|c|c|c|c|}
\hline Cells & $w_{m i}$ & $w_{c i}$ & Cells & $w_{m i}$ & $w_{c i}$ & Cells & $w_{m i}$ & $w_{c i}$ & Cells & $w_{m i}$ & $w_{c i}$ \\
\hline 1 & 553 & 968 & 17 & 626 & 184 & 33 & 121 & 952 & 49 & 524 & 345 \\
\hline 2 & 907 & 745 & 18 & 104 & 787 & 34 & 1410 & 367 & 50 & 1400 & 135 \\
\hline 3 & 515 & 827 & 19 & 1408 & 319 & 35 & 1011 & 132 & 51 & 393 & 175 \\
\hline 4 & 1965 & 705 & 20 & 1256 & 25 & 36 & 1298 & 439 & 52 & 1272 & 596 \\
\hline 5 & 1336 & 902 & 21 & 1637 & 934 & 37 & 1634 & 134 & 53 & 1197 & 677 \\
\hline 6 & 1318 & 498 & 22 & 1950 & 414 & 38 & 1750 & 153 & 54 & 462 & 283 \\
\hline 7 & 1292 & 807 & 23 & 101 & 104 & 39 & 1948 & 612 & 55 & 548 & 139 \\
\hline 8 & 1789 & 62 & 24 & 539 & 881 & 40 & 662 & 216 & 56 & 500 & 307 \\
\hline 9 & 541 & 331 & 25 & 655 & 694 & 41 & 700 & 878 & 57 & 113 & 272 \\
\hline 10 & 1071 & 212 & 26 & 131 & 793 & 42 & 765 & 957 & 58 & 47 & 931 \\
\hline 11 & 1759 & 787 & 27 & 1227 & 955 & 43 & 756 & 363 & 59 & 1676 & 38 \\
\hline 12 & 1416 & 664 & 28 & 450 & 126 & 44 & 436 & 820 & 60 & 1017 & 896 \\
\hline 13 & 1413 & 938 & 29 & 470 & 268 & 45 & 672 & 362 & 61 & 1307 & 164 \\
\hline 14 & 1224 & 719 & 30 & 1081 & 96 & 46 & 822 & 356 & 62 & 499 & 78 \\
\hline 15 & 484 & 794 & 31 & 1714 & 285 & 47 & 1912 & 637 & 63 & 1451 & 303 \\
\hline 16 & 543 & 543 & 32 & 308 & 368 & 48 & 1402 & 626 & 64 & 1606 & 578 \\
\hline
\end{tabular}

TABLE 6: Results for the $8 \times 8$ network.

\begin{tabular}{|c|c|c|c|c|c|c|}
\hline Algorithms & Average value & Deviation & Minimum value & Deviation & Maximum value & Deviation \\
\hline GA [26] & 14.005 & $1.619 \%$ & 13.782 & $0.000 \%$ & 14.617 & $6.454 \%$ \\
\hline TS [26] & 13.791 & $0.071 \%$ & 13.782 & $0.000 \%$ & 13.999 & $1.580 \%$ \\
\hline $\mathrm{AC}[26]$ & 14.107 & $2.361 \%$ & 13.801 & $0.141 \%$ & 14.407 & $4.539 \%$ \\
\hline TDE [27] & 13.889 & $0.776 \%$ & 13.782 & $0.000 \%$ & 14.093 & $2.256 \%$ \\
\hline HCDE [27] & 13.788 & $0.043 \%$ & 13.782 & $0.000 \%$ & 13.892 & $0.798 \%$ \\
\hline \multicolumn{7}{|c|}{ Our methods } \\
\hline GSO & 13.791 & $0.497 \%$ & 13.782 & $0.000 \%$ & 14.102 & $2.321 \%$ \\
\hline DGSO & 13.780 & $0.037 \%$ & 13.782 & $0.000 \%$ & 13.883 & $0.786 \%$ \\
\hline
\end{tabular}

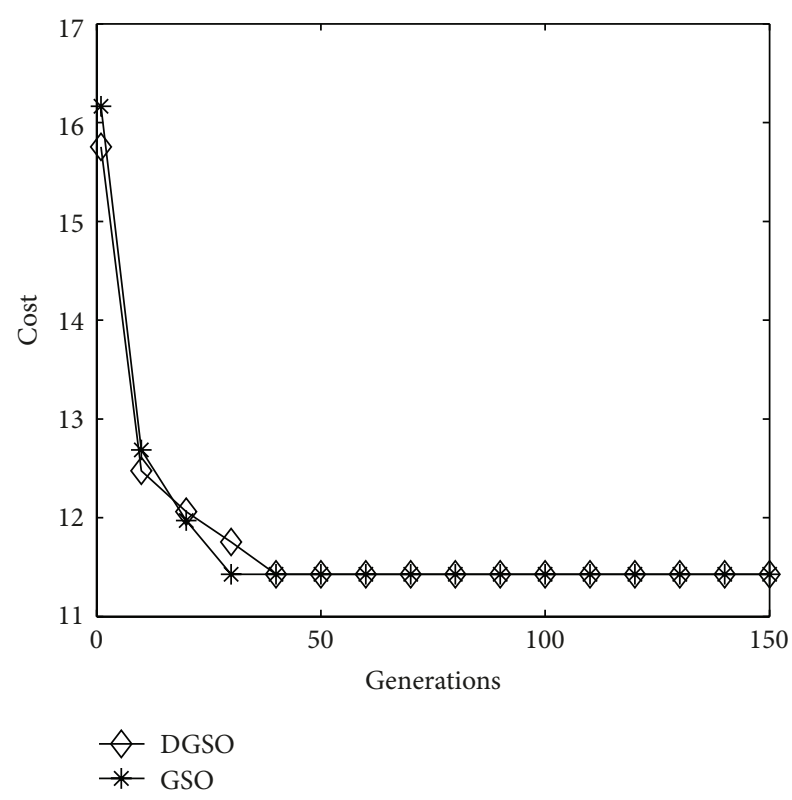

FIgURE 5: Performance index in successive generations $(6 \times 6$ network).
4.3. Test-Network with 64 Cells. The last experiment is carried out on a $8 \times 8$ size test network, whose data set is given in Table 5 [26]. Figure 7 describes the process of optimization using GSO and DGSO with a size of 100 populations and 150 generations, respectively. It shows that DGSO has less cost and rapid convergence in comparison with GSO. In the $4 \times 4$ and $6 \times 6$ size test networks, the results of DGSO are the same as the GSO due to the relative small size of test network. With the increase of the size of network, we can safely expect that the DGSO may obtain better performance than the original GSO.

The cost of the proposed DGSO is also compared with the performance of some other algorithms; refer to Table 6. The minimum value of the DGSO and some other algorithms are almost the same; however, it is evident that the performance of the proposed DGSO is better in sense of its maximum value and deviation. Figure 8 describes the optimal solution with reporting cells configuration.

\section{Conclusions}

This paper presents the DGSO and shows its application to mobile location management problem. Two important aspects are worth highlighting here. 


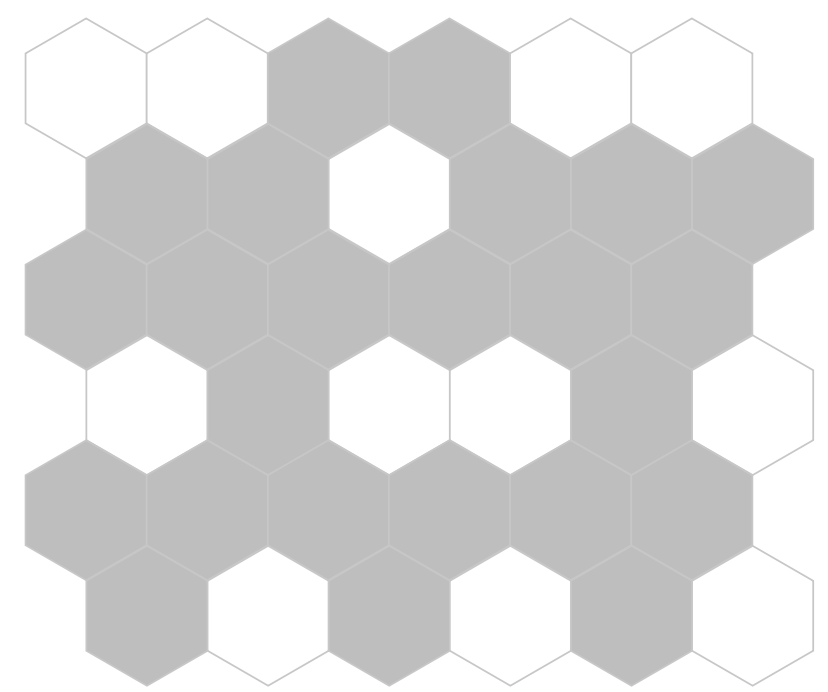

FIGURE 6: $6 \times 6$ test network optimal solution with reporting cells configuration.

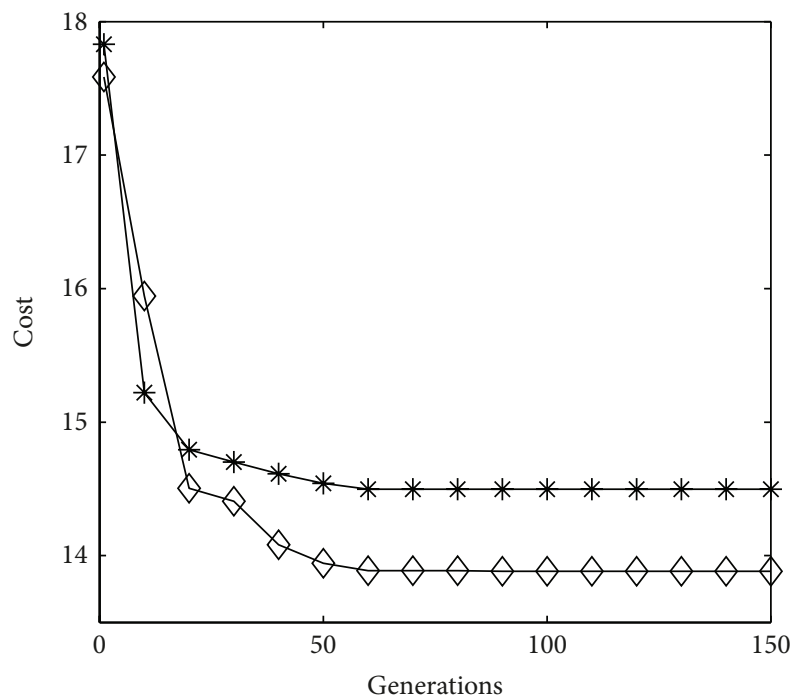

$\diamond$ DGSO

* GSO

Figure 7: Performance index in successive generations $(8 \times 8$ network).

(1) We proposed the design of DGSO with the aid of diversity operator. Experimental results based on $8 \times 8$ test network demonstrate the contribution of diversity-guided operator.

(2) We employ GSO and DGSO to deal with the mobile location management problem. Experimental results show that DGSO exhibits good performance in the comparison with some other algorithms reported in the literatures.

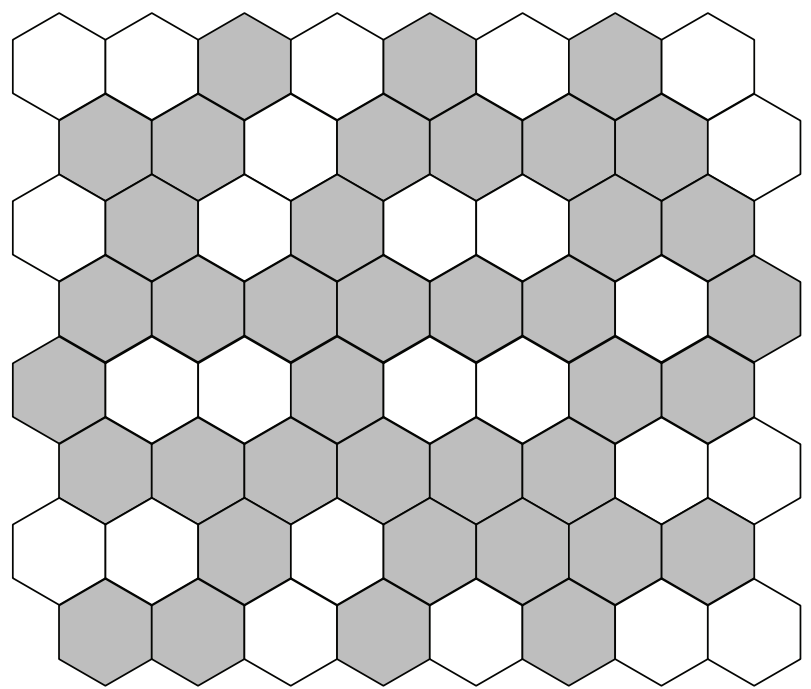

FIGURE 8: $8 \times 8$ test network optimal solution with reporting cells configuration.

\section{Conflict of Interests}

The authors declare that there is no conflict of interests regarding the publication of this paper.

\section{Acknowledgments}

This work was supported by the National Natural Science Foundation of China (Grant no. 61272509 and Grant no. 61301140) and supported by the Open Funding Project of State Key Laboratory of Virtual Reality Technology and Systems, Beihang University, China (Grant no. BUAA-VR14KF-11).

\section{References}

[1] R. Subrata and A. Y. Zomaya, "Evolving cellular automata for location management in mobile computing networks," IEEE Transactions on Parallel and Distributed Systems, vol. 14, no. 1, pp. 13-26, 2003.

[2] Y. B. Lin and I. Chlamatac, Wireless and Mobile Network Architecture, John Wiley \& Sons, London, UK, 2001.

[3] V. W. S. Wong and V. C. M. Leung, "Location management for next-generation personal communications networks," IEEE Network, vol. 14, no. 5, pp. 18-24, 2000.

[4] Q. Zeng and D. P. Agrawal, "Performance analysis of a handoff scheme in integrated voice/data wireless networks," in Proceedings of the 52nd IEEE VTS Fall Vehicular Technology Conference (VTC '00), pp. 1986-1992, September 2000.

[5] S. M. Almeida-Luz, M. A. Vega-Rodríguez, J. A. Gómez-Púlido, and J. M. Sánchez-Pérez, "Differential evolution for solving the mobile location management," Applied Soft Computing Journal, vol. 11, no. 1, pp. 410-427, 2011.

[6] S. Kim, G. Kim, J. Byeon, and J. Taheri, "Particle swarm optimization for location mobility management," International Journal of Innovative Computing, Information and Control, vol. 8, no. 12, pp. 8387-8398, 2012. 
[7] J. Taheri and A. Y. Zomaya, "A Simulated Annealing approach for mobile location management," Computer Communications, vol. 30, no. 4, pp. 714-730, 2007.

[8] J. Taheri and A. Y. Zomaya, "Realistic simulations for studying mobility management problems," International Journal of Wireless and Mobile Computing, vol. 1, no. 8, 2005.

[9] P. Demestichas, N. Georgantas, E. Tzifa et al., "Computationally efficient algorithms for location area planning in future cellular systems," Computer Communications, vol. 23, no. 13, pp. 12631280, 2000.

[10] W. H. A. Yuen and W. S. Wong, "A dynamic location area assignment algorithm for mobile cellular systems," in Proceedings of the IEEE International Conference on Communications (ICC '98), vol. 3, pp. 1385-1389, Atlanta, Ga, USA, June 1998.

[11] S. He, Q. H. Wu, and J. R. Saunders, "A novel group search optimizer inspired by animal behavioural ecology," in Proceedings of the IEEE Congress on Evolutionary Computation (CEC '06), pp. 1272-1278, Canada, July 2006.

[12] G. S. Sharvani, N. K. Cauvery, and T. M. Rangaswamy, "Different types of swarm intelligence algorithm for routing," in Proceedings of the International Conference on Advances in Recent Technologies in Communication and Computing (ARTCom '09), pp. 604-609, Kottayam, India, October 2009.

[13] X. P. Du, L. Z. Cheng, and L. F. Liu, "A swarm intelligence algorithm for joint sparse recovery," IEEE Signal Processing Letters, vol. 20, no. 6, pp. 611-614, 2013.

[14] A. Y. Zomaya and R. Kazman, "Simulated annealing techniques," in The handbook of Algorithms and Theory of Computation, M. J. Atallah, Ed., pp. 37.1-37.19, CRC Press, Boca Raton, Fla, USA, 1999.

[15] J. Taheri and A. Y. Zomaya, "A genetic algorithm for finding optimal location area configurations for mobility management," in Proceedings of the 30th Anniversary of the IEEE Conference on Local Computer Networks (LCN '05), pp. 568-577, Sydney, Australia, November 2005.

[16] J. Taheri and A. Y. Zomaya, "The use of a Hopfield neural network in solving the mobility management problem," in Proceedings of the IEEE/ACS International Conference on Pervasive Services (ICPS '04), pp. 141-150, Beirut, Lebanon, July 2004.

[17] A. Abraham, R. K. Jatoth, and A. Rajasekhar, "Hybrid differential artificial bee colony algorithm," Journal of Computational and Theoretical Nanoscience, vol. 9, no. 2, pp. 249-257, 2012.

[18] S. He, Q. H. Wu, and J. R. Saunders, "Group search optimizer: an optimization algorithm inspired by animal searching behavior," IEEE Transactions on Evolutionary Computation, vol. 13, no. 5, pp. 973-990, 2009.

[19] C. J. Barnard and R. M. Sibly, "Producers and scroungers: a general model and its application to captive flocks of house sparrows," Animal Behaviour, vol. 29, no. 2, pp. 543-550, 1981.

[20] D. Mustard, "Numerical integration over the $n$-dimensional spherical shell," Mathematics of Computation, vol. 18, pp. 578$589,1964$.

[21] W. J. O’Brien, B. I. Evans, and G. L. Howick, "A new view of the predation cycle of a planktivorous fish, white crappie (Pomoxis annularis)," Canadian Journal of Fisheries and Aquatic Sciences, vol. 43, no. 10, pp. 1894-1899, 1986.

[22] G. M. Viswanathan, S. V. Buldyrev, S. Havlin, M. G. E. Da Luz, E. P. Raposo, and H. E. Stanley, "Optimizing the success of random searches," Nature, vol. 401, no. 6756, pp. 911-914, 1999.

[23] K. Li, "Performance analysis and evaluation of random walk algorithms on wireless networks," in Proceedings of the IEEE
International Symposium on Parallel and Distributed Processing, Workshops and Phd Forum (IPDPSW '10), pp. 1-8, Atlanta, Ga, USA, April 2010.

[24] H. Ono, "Fast random walks on finite graphs and graph topological information," in Proceedings of the 2nd International Conference on Networking and Computing (ICNC '11), pp. 360363, Osaka, Japan, December 2011.

[25] J. Riget and J. S. Vesterstrøm, "A diversity-guided particle swarm optimizer-the ARPSO," Tech. Rep., EVAlife, Copenhagen, Denmark, 2002.

[26] R. Subrata and A. Y. Zomaya, "A comparison of three artificial life techniques for reporting cell planning in mobile computing," IEEE Transactions on Parallel and Distributed Systems, vol. 14, no. 2, pp. 142-153, 2003.

[27] W. Wang, F. Wang, Q. Pan, and F. Zuo, "Improved differential evolution algorithm for location management in mobile computing," in Proceedings of the International Workshop on Intelligent Systems and Applications (ISA '09), pp. 1-5, Wuhan, China, May 2009. 

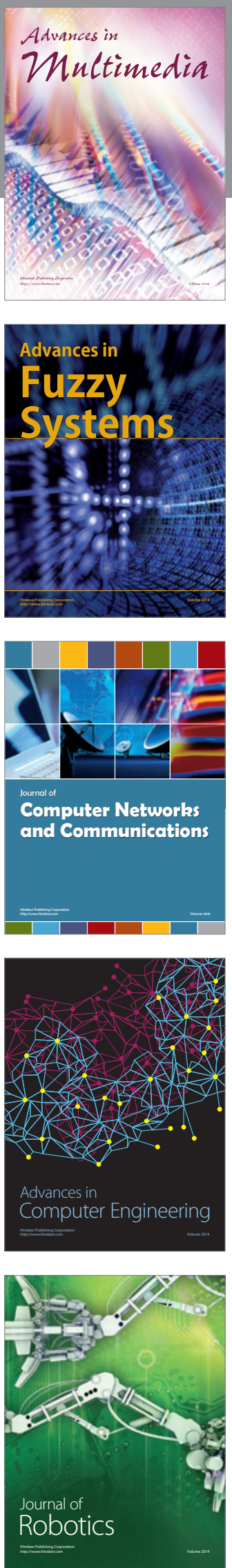

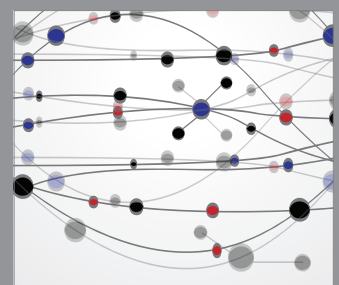

The Scientific World Journal
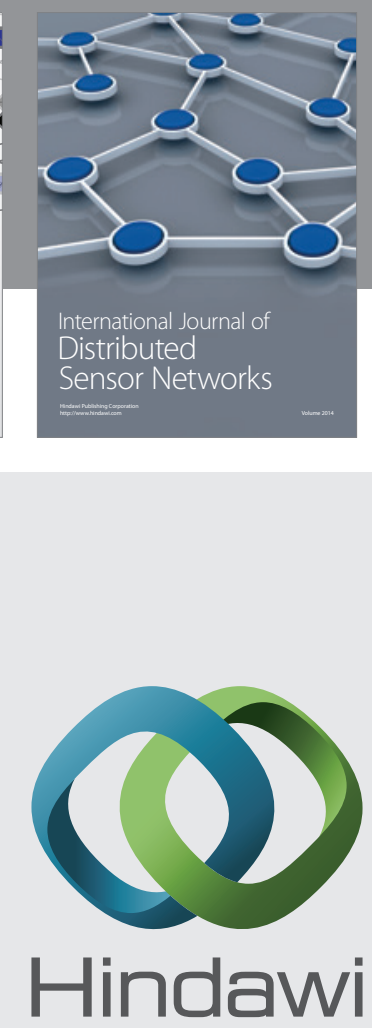

Submit your manuscripts at

http://www.hindawi.com
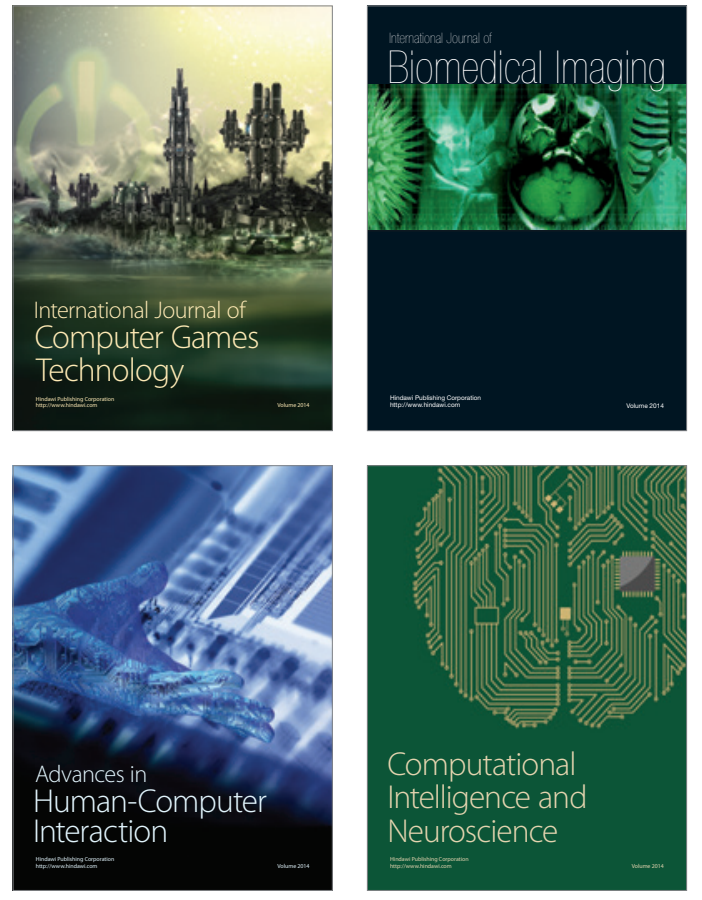
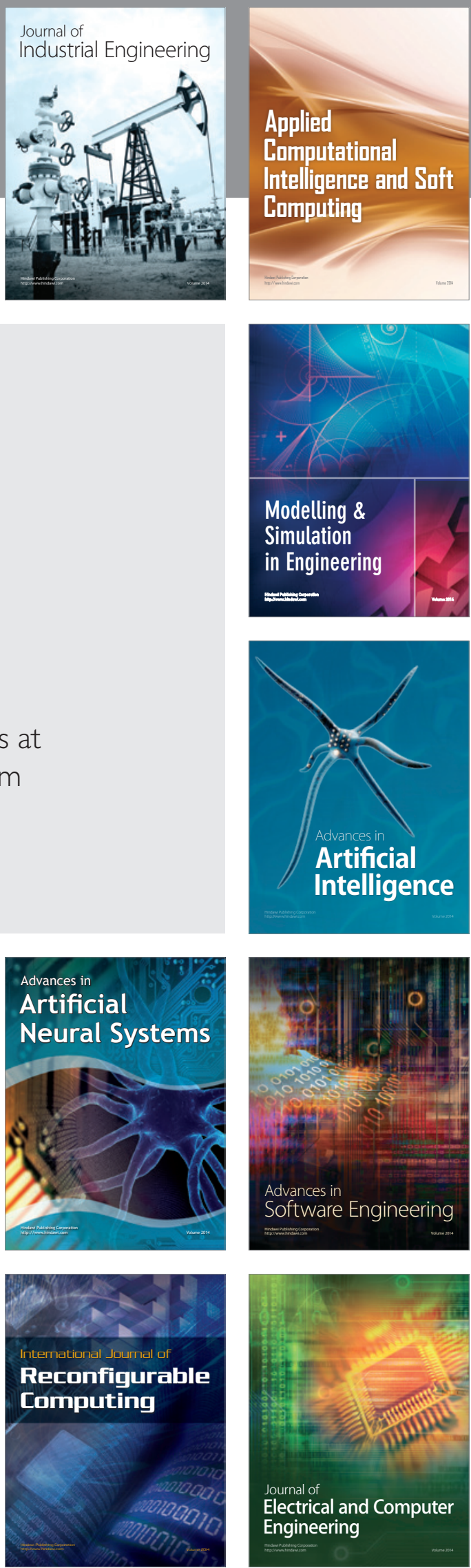